\title{
MODELLING AND OPTIMIZATION FOR A SELECTIVE ASSEMBLY PROCESS OF PARTS WITH NON-NORMAL DISTRIBUTION
}

\author{
Wang, W. M.; Li, D. B.\#; He, F. \& Tong, Y. F. \\ School of Mechanical Engineering, Nanjing University of Science and Technology, Nanjing, China \\ E-Mail: w.m.wang@ njust.edu.cn ( ${ }^{\#}$ Corresponding author)
}

\begin{abstract}
Selective assembly method has progressed significantly for the past few years to become a valuable tool for improving quality in product assembly process where the required assembly precision is very high. In traditional selective assembly process, if the mating parts with non-normal distribution are grouped and assembled, many assembled products fail to meet the assembly precision requirement and thereby being identified as unacceptable to be scrapped. This paper proposes an approach by applying improved grouping selective assembly scheme to a ball-bearing assembly, to reduce the surplus parts and hence to improve acceptance rate of assembled products. A solving algorithm is presented based on genetic algorithm (GA), where the elitist strategy is integrated to improve the convergence of the algorithm, and the simulation is utilized to give better insight into the optimization process. Finally, some numerical experiments in different cases are conducted, which demonstrate that the proposed approach outperforms traditional selective assembly method in generating solutions with maximum assembly acceptance rate.

(Received, processed and accepted by the Chinese Representative Office.)
\end{abstract}

Key Words: Selective Assembly Process, Grouping Scheme, Modelling, Optimization

\section{INTRODUCTION}

Due to increasing competition in the developing global manufacturing environment, the required product's complexity and technical level push manufacturing industry towards higher precision in upstream manufacturing phases as well as downstream assembly process. Nevertheless, variation is unavoidable in any manufacturing processes: although parts and assemblies are supposed to be identical, they often differ from what they are expected to be. In fact, with respect to the key characteristic of each part, the actual value will deviate from the expected value. Consequently, in assembly process, variation of manufactured parts will accumulate through dimensional chains that pass through the parts. Specially, for assemblies with tight tolerances, the manufacturing processes of the parts are either not able to satisfy the precision requirement or only at high cost [1].

To cope with this challenge, the concept of selective assembly is proposed, which is a preferred method to achieve a high-precision assembly from compatible parts of relatively low precision. Thus, this method allows either to increase the final product quality or to decrease the production cost to satisfy the customer's requirement. With the rapid development of high-end computers and advanced measurement technologies in the past decade years, selective assembly technology has been commonly applied in many industries, especially in high precision manufacturing sectors such as micro-production [2], hard disk drives [3], batteries assembly [4], battery electrodes [5], and in the automotive body assembly [6].

In conventional selective assembly process, parts are grouped with equal width or with equal probability, and then matched based on their grouping number. The dimensional distributions of parts are all similar and normal in the majority of relevant research cases. These methods are only suitable for addressing the assembly problems in mass production. 
However, in view of high-precision assembles in low-volume production, the parts are usually non-normally distributed. Therefore, from the perspectives of both economy and quality, the main purpose in this work is to provide a cost-effective grouping scheme in selective assembly process for parts with non-normal distribution which can decrease the surplus parts in order to increase the acceptance rate of assembled products.

The rest of this article is organized as follows. In Section 2, the existing literature is reviewed from the aspects of reducing surplus parts and minimizing assembly variation in selective assembly process. Section 3 states the research problem in detail. In this section, we propose an improved grouping selective assembly scheme for ball-bearing assembly products based on the new grouping coding scheme. Moreover, the formulation of mathematical model is presented in this section. The improved genetic algorithm to find the optimal assembly scheme is presented in Section 4. In Section 5, we conduct some numerical experiments of selective assembly process for ball-bearing assembly products to illustrate the competitive advantage of the proposed approach. Finally, the conclusion is drawn in Section 6.

\section{LITERATURE REVIEW}

By applying selective assembly method, most researches on assembly quality improvement have been focused on reducing surplus parts and minimizing assembly variation. The relevant literatures are reviewed in this section.

In terms of reducing the surplus parts in selective assembly process, some approaches have been proposed. Fang and Zhang [7] proposed a new grouping method with equal probability. The mating parts were manufactured within the designed tolerance specifications and the grouping process was conducted after manufacturing. In the proposed method, the surplus parts were reduced in a large part in comparison with traditional method. Kumar et al. [8] suggested an effective two-stage approach. In the first stage, the surplus parts were produced under an equal width grouping scheme. In the second stage, the surplus parts were classified into three groups. The optimal solution for the grouping combination was obtained by using genetic algorithm. Matsuura and Shinozaki [9] proposed an approach of optimal process design in selective assembly process at three shifted means. In the proposed approach, the surplus parts were minimized under equal width grouping schemes. Raj et al. [10] introduced a new batch selective assembly approach for a ball bearing assembly. In this proposed approach, the surplus parts were minimised and the manufacturing system efficiency was maximised. Lu and Fei [11] proposed an improved selective assembly method to minimise the number of surplus parts by using genetic algorithm. In consideration of different tolerance ranges of parts, a new grouping method was suggested and the effect on the assembly success rate for each group was investigated.

Moreover, several scholars conduct their researches on minimizing the assembly variation in selective assembly process. Mease et al. [12] defined that selective assembly was a costbenefit method to reduce the assembly variation and hence to improve the quality of a final product. Kannan et al. [13] improved the assembly complexity of product with a third part. A particle swarm optimization algorithm was developed to match the compatible parts of tolerance groups in order to minimise the assembly variation of the final product. Wang et al. [14] suggested an improved method for a selective assembly process of parts with similar or dissimilar dimensional distribution. In this method, the best combinations of groups were found by using genetic algorithm in order to achieve the minimum assembly clearance variation. Raj et al. [15] suggested an improved batch selective assembly approach for complex assembly with three parts. In this approach, genetic algorithm was applied to obtain the best combination of parts in order to minimise the assembly variation and maximise the manufacturing system efficiency. 
In summary, it can be found that the current researches have focused mainly on the optimization of grouping selective assembly process for parts with normally distributed. However, very few researches have been focused on the optimization of grouping selective assembly process for parts with non-normally distributed. Therefore, with respect to the parts with non-normally distributed, which are the cases in the majority of manufacturing industries, our research in this article aims at filling in that blank and addresses the problem of grouping selective assembly optimization in the ball-bearing assembly process.

\section{MODEL DEVELOPMENT}

\subsection{Background of the problem}

Linear assembly for parts of single dimensional chain, which is the basis of complex assembly, is one of the hottest issues in the product assembly field at present. In this study, we consider a product of ball-bearing assembly with three types of parts: outer race, ball and inner race, as illustrated in Fig. 1. The fit of ball-bearing assembly is clearance fit. In order to make the bearing rotated easily and avoid noisy operation, the designed assembly clearance requirement is $0_{+0.018}^{+0.024} \mathrm{~mm}$.

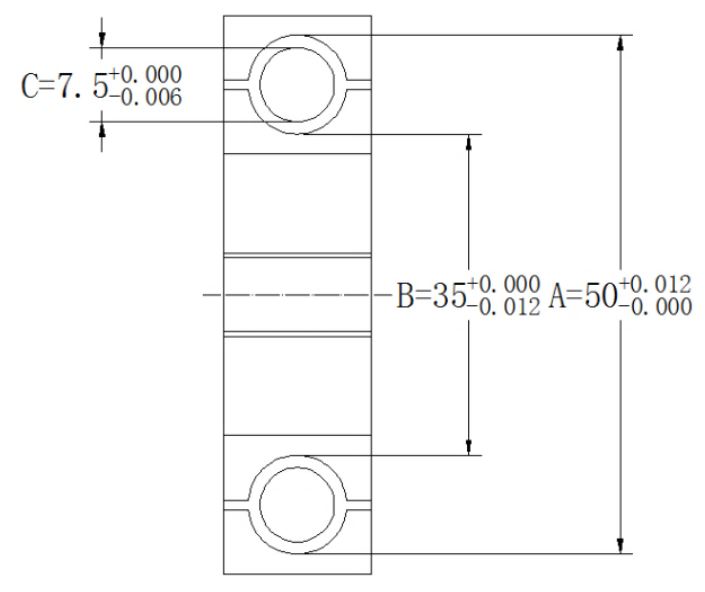

Figure 1: Ball-bearing assembly.

The design tolerance specifications for ball-bearing assembly are tabulated in Table I. The mating parts are mass manufactured through different processes in different machines. In general, the dimensions of parts in mass production follow normal distribution. However, when the volume of parts is divided into lower volumes, the parts in each volume are all nonnormal distributed through Anderson-Darling test [16]. The dimension tolerances of parts A$\mathrm{C}$ of 50 parts, which comes from literature [17], are given in Table II. Under the high assembly precision requirement, the ball-bearing assembly process has the following problems.

(1) Low fraction of parts to be assembled: If the parts with non-normal distribution are grouped as in traditional selective assembly method, the numbers of parts in matching groups are very different. Accordingly, many matching parts are not going to be assembled, i.e., the fraction of parts to be assembled is very low.

(2) High assembly dimensional variations: As shown in Fig. 1, if all the mating parts are assembled randomly and interchangeably, the assembly clearance is $(A-B)-2 * C=$ $\left(50_{-0.000}^{+0.012}-35_{-0.012}^{+0.000}\right)-\left(2 \times 7.5_{-0.006}^{+0.000}\right)=0_{-0.000}^{+0.036} \mathrm{~mm}$, namely the assembly clearance variation is $36 \mu \mathrm{m}$, which is very high.

Due to the reasons as mentioned above, if ball-bearing assembly products are produced through low precision parts in a large volume, many assembled products are not able to meet 
the assembly clearance requirement, i.e., the assembly acceptance rate is particularly low. In order to address this problem, a new grouping selective assembly scheme is proposed by using a new grouping coding method and an improved genetic algorithm. The detailed description for the new scheme is presented in the following sections.

Table I: Design tolerance specification for ball-bearing assembly.

\begin{tabular}{|l|l|c|c|}
\hline \multicolumn{1}{|c|}{ Part } & Dimension characteristic & Part tolerance & Assembly requirement \\
\hline Outer race & Abutment diameter (A) & $50_{-0.000}^{+0.012} \mathrm{~mm}$ & \multirow{2}{*}{$0_{+0.018}^{+0.024} \mathrm{~mm}$} \\
\hline Inner race & Abutment diameter (B) & $35_{-0.012}^{+0.000} \mathrm{~mm}$ & \\
\hline Ball & Diameter (C) & $7.5_{-0.006}^{+0.000} \mathrm{~mm}$ & \\
\hline
\end{tabular}

Table II: Dimension tolerances data of example parts.

\begin{tabular}{|c|c|}
\hline Part type & Individual part tolerances ( $\mu \mathrm{m}$, from no. 1 to no. 50 ) \\
\hline A & $\begin{array}{l}5,3,6,7,4,8,8,6,7,6,6,7,5,10,6,6,8,6,6,4,7,3,7,9,5,8,9,3,3,7,5,7,8,7,9,7,8,4,6,6,3,7,4,9,4,7,6 \\
4,2,6\end{array}$ \\
\hline B & $\begin{array}{l}-8,-9,-9,-4,-7,-8,-4,-6,-8,-3,-4,-8,-6,-7,-9,-7,-4,-8,-6,-4,-10,-6,-4,-7,-9,-7,-8,-6,-6,-8,-6,-6, \\
-5,-5,-5,-4,-6,-6,-6,-2,-5,-6,-7,-6,-6,-3,-7,-6,-7,-7\end{array}$ \\
\hline $\mathrm{C}$ & $\begin{array}{l}-2,-3,-3,-2,-4,-3,-4,-2,-2,-3,-3,-5,-3,-5,-5,-1,-1,-2,-3,-3,-4,-2,-2,-3,-3,-3,-4,-4,-1,-4,-4,-4,-4, \\
-3,-2,-3,-1,-3,-3,-2,-2,-2,-3,-3,-3,-3,-3,-3,-2,-4\end{array}$ \\
\hline
\end{tabular}

\subsection{Grouping selective assembly scheme coding rules}

\section{Calculation for grouping number}

According to the parts tolerance distribution of and the assembly tolerance requirement, the number of groups in parts grouping can be determined. Compared through calculation the number of groups is obtained using the following equation:

$$
k=\left\lceil\frac{T_{A}+T_{B}+2 T_{C}}{T_{\sigma}}\right\rceil
$$

where $k$ is the number of groups. $T_{A}, T_{B}$, and $T_{C}$ are the design tolerance width of part A, part $\mathrm{B}$ and part $\mathrm{C}$ in an assembly, respectively. $T_{\sigma}$ represents the required assembly tolerance width. The square brackets on the right side of equal sign means rounding up to the nearest integer.

As mentioned in Table I, in ball-bearing assembly, the design tolerance width of out race, inner race and ball are $12 \mu \mathrm{m}, 12 \mu \mathrm{m}$ and $6 \mu \mathrm{m}$, and the tolerance width of assembly requirement is $6 \mu \mathrm{m}$. The number of groups $k$ is obtained based on the Eq. (1). It is shown as below:

$$
k=\left\lceil\frac{12+12+2 \times 6}{6}\right\rceil=6
$$

\section{Coding for grouping scheme on tolerance range}

The grouping scheme on tolerance range is described by a sequence of floating point numbers which represent the relative width of grouping tolerance range, as show below:

$$
\begin{gathered}
{\left[\begin{array}{ccccc}
r_{1,1} & r_{1,2} & r_{1,3} & \cdots & r_{1, k} \\
r_{2,1} & r_{2,2} & r_{2,3} & \cdots & r_{2, k} \\
r_{3,1} & r_{3,2} & r_{3,3} & \cdots & r_{3, k} \\
\vdots & \vdots & \vdots & \ddots & \vdots \\
r_{m, 1} & r_{m, 2} & r_{m, 3} & \cdots & r_{m, k}
\end{array}\right]_{m \times k}} \\
\\
r_{i, j}=t_{i, j} / \sum_{j=1}^{k} t_{i, j}
\end{gathered}
$$


where $m$ means the number of part types, $k$ is the number of groups, $t_{i, j}$ is the width of tolerance range on the $j^{\text {th }}$ group of the $i^{\text {th }}$ type of parts. For instance, for the first row of the chromosome, i.e., for the first type of parts (outer race), the diameter tolerance range is [0 12] $\mu \mathrm{m}$, the tolerance width is $12 \mu \mathrm{m}$, and a gene sequence is $\left[\begin{array}{lll}0.1263 & 0.18180 .0599 & 0.2087\end{array}\right.$ $0.29700 .1264]$. The width of tolerance range on every group is $12 * 0.1263 \mu \mathrm{m}, 12 * 0.1818 \mu \mathrm{m}$, $12 * 0.0599 \mu \mathrm{m}, 12 * 0.2087 \mu \mathrm{m}, 12 * 0.2970 \mu \mathrm{m}, 12 * 0.1264 \mu \mathrm{m}$, successively.

\section{Coding for grouping matching scheme}

The matching relationship among groups is coded by natural number coding method. For the permutation of all types of parts in a matching scheme, the structure of a matrix is shown as follows:

$$
\left[\begin{array}{ccccc}
s_{1,1} & s_{1,2} & s_{1,3} & \cdots & s_{1, k} \\
s_{2,1} & s_{2,2} & s_{2,3} & \cdots & s_{2, k} \\
s_{3,1} & s_{3,2} & s_{3,3} & \cdots & s_{3, k} \\
\vdots & \vdots & \vdots & \ddots & \vdots \\
s_{m, 1} & s_{m, 2} & s_{m, 3} & \cdots & s_{m, k}
\end{array}\right]_{m \times k}
$$

where $m$ means the number of part types, $k$ is the number of groups, $\left[\begin{array}{lllll}s_{i, 1} & s_{i, 2} & s_{i, 3} & \cdots & s_{i, k}\end{array}\right]$ represents the permutation of group number set $\{1,2,3, \cdots, k\}$ in the $i^{\text {th }}$ type of parts. For instance, a gene matrix is $\left[\begin{array}{l}415623 \\ 531246 \\ 642351\end{array}\right], m=3, k=6$, as mentioned above. It means that the fourth group of part A, the fifth group of part B and sixth group of part $\mathrm{C}$ should be matched together, the first group of part A, the third group of part B and fourth group of part C should be matched together, and so on.

Finally, considering that the genes of grouping scheme on tolerance range and grouping matching scheme are floating point numbers and integers respectively, a simple addition operation on the two gene matrixes is made to facilitate the subsequent related operations. Thus, the structure of chromosome is shown as follows:

$$
\text { Chrom }_{i}=\left[\begin{array}{ccccc}
r_{1,1}+s_{1,1} & r_{1,2}+s_{1,2} & r_{1,3}+s_{1,3} & \cdots & r_{1, k}+s_{1, k} \\
r_{2,1}+s_{2,1} & r_{2,2}+s_{2,2} & r_{2,3}+s_{2,3} & \cdots & r_{2, k}+s_{2, k} \\
r_{3,1}+s_{3,1} & r_{3,2}+s_{3,2} & r_{3,3}+s_{3,3} & \cdots & r_{3, k}+s_{3, k} \\
\vdots & \vdots & \vdots & \ddots & \vdots \\
r_{m, 1}+s_{m, 1} & r_{m, 2}+s_{m, 2} & r_{m, 3}+s_{m, 3} & \cdots & r_{m, k}+s_{m, k}
\end{array}\right]_{m \times k}
$$

In order to ensure that the generated solution is feasible, the chromosome structure must satisfy the following constrains in Eq. (7):

$$
\left\{\begin{array}{l}
\sum_{j=1}^{k} r_{i, j}=1 \\
\bigcup_{i=1}^{m}\{1,2,3, \cdots, k\}-\left\{s_{i, 1}, s_{i, 2}, s_{i, 3}, \cdots, s_{i, k}\right\}=\varnothing
\end{array}\right.
$$

\subsection{Formulation of optimization objective in grouping selective assembly process}

In ball-bearing assembly process, there are three main objectives: assembly precision $P$, production cost $C$ and assembly acceptance rate $R$. In the traditional methods of grouping selective assembly, minimising the assembly clearance variation is generally identified as the optimization objective for improving the assembly precision and reducing the surplus parts simultaneously. However, the assembly precision requirement is generally given in the design phase, so it is not necessary to further minimise the assembly clearance variation as long as the final assembly precision meets specification. Instead of considering production $\operatorname{cost} C$, all 
the parts involved in grouping selective assembly are mass production, so the final total parts cost of each product is fixed.

Moreover, grouping management on parts will lead to additional cost. In the original operation design, parts are numbered and put in storage prior to assembly operation, meanwhile the part characteristics are measured and recorded. In fact, a unified method for measuring characteristics of parts with grouping management can improve the measurement efficiency and reduce the amount of numbering work, and the additional cost from grouping management can be offset. Thus, the influence of this factor can be ignored.

We consider $F$ and $S$ as our objective function and grouping selective assembly scheme respectively. The optimization objective can be stated as follows:

$$
\max F(S)=R(S)
$$

\section{THE IMPROVED GA FOR SELECTIVE ASSEMBLY PROCESS}

According to the proposed grouping coding scheme and optimization model, it is found that a ball-bearing assembly with $m$ types and $k$ groups of parts offers more than $6^{m \times k}$ possible assembly combinations. Comparing the assembly acceptance rates for all possible combinations which are generated via complete enumeration method is too time-consuming to be feasible. Hence, it is more efficient to apply meta-heuristic methods, which take limited computation time to obtain nearly optimal solutions. Some commonly used algorithms (e.g., simulated annealing algorithm, genetic algorithm, particle swarm optimization, ant colony algorithm), can be used to solve many problems, such as bulk cargo port scheduling [18], production coordination [19], electric vehicle charging station location [20], and so on [2123]. In this research project, an improved GA is developed to identify the optimal assembly scheme. The framework of this algorithm is illustrated in Fig. 2, and its detailed design layout is presented in the following sections.

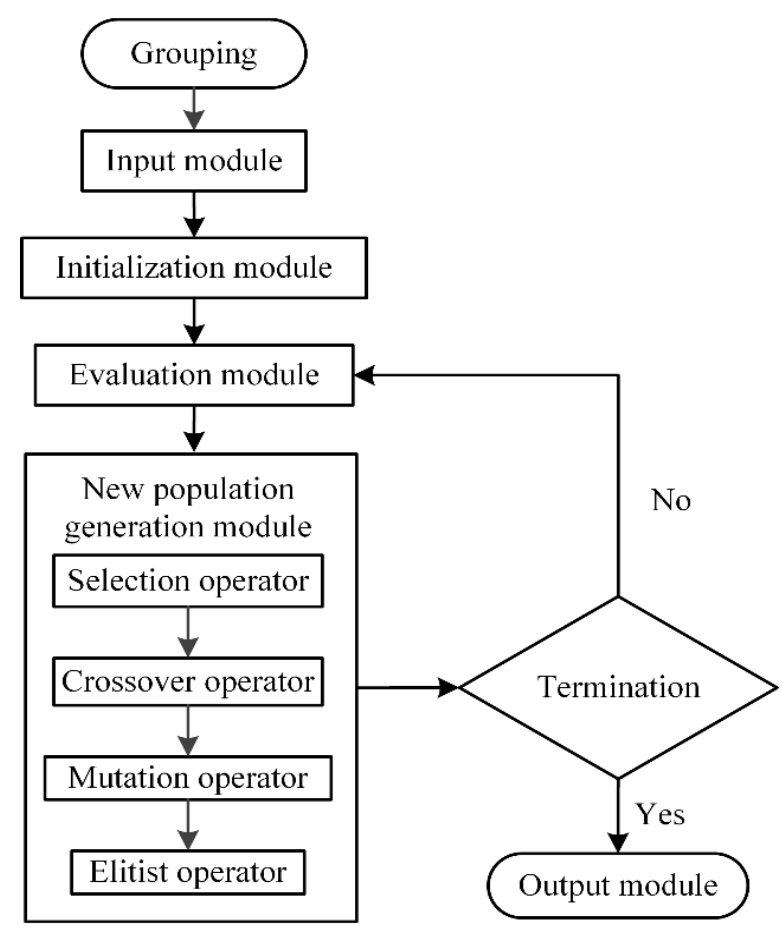

Figure 2: Framework of the improved GA.

\subsection{Input module}

In this module, the required data are: 
(1) Tolerance range of parts

(2) Number of part types: $m$

(3) Number of parts in a volume: $n$

(4) Assembly precision requirement: $T_{\sigma}$

(5) Grouping data of parts

(6) Size of population: $N$

(7) Probability of crossover: $P_{c}$

(8) Probability of mutation: $P_{m}$

To better demonstrate the overall process of the proposed algorithm, a numerical example is used by inputting the following data:

(1) Shown in Table I

(2) $m=3$

(3) $n=50$

(4) $T_{\sigma}=\left[T_{\min }, T_{\max }\right]=[18,24]$

(5) Shown in Table III

(6) $N=10$

(7) $P_{c}=0.9$

(8) $P_{m}=0.05$

Table III: Grouping data of example parts.

\begin{tabular}{|l|c|c|c|c|c|c|}
\hline \multicolumn{1}{|c|}{ Part \Group no. } & 1 & 2 & 3 & 4 & 5 & 6 \\
\hline Tolerance range & {$[0,2.1]$} & $(2.1,3.6]$ & $(3.6,5.8]$ & $(5.8,6.8]$ & $(6.8,9.1]$ & $(9.1,12]$ \\
\hline Number of part A & 1 & 5 & 10 & 12 & 21 & 1 \\
\hline Tolerance range & {$[-12,-11]$.} & $(-11.1,-10.9]$ & $(-10.9,-7.6]$ & $(-7.6,-5.7]$ & $(-5.7,-3.1]$ & $(-3.1,0]$ \\
\hline Number of part B & 0 & 0 & 12 & 24 & 11 & 3 \\
\hline Tolerance range & {$[-6,-5.9]$} & $(-5.9,-3.2]$ & $(-3.2,-2.7]$ & $(-3.2,-2.7]$ & $(-2.7,-1.8]$ & $(-1.8,0]$ \\
\hline Number of part C & 0 & 13 & 21 & 12 & 0 & 4 \\
\hline
\end{tabular}

\subsection{Initialization module}

Based on the coding style in section 3.2, the initial population size $N$ is set as 10 . The initial population Pop $=\left\{\right.$ chrom $_{1}$, chrom $_{2}$, chrom $_{3}, \cdots$, chrom $\left._{10}\right\}$ is generated randomly in order to guarantee that each individual in the initial population is distributed in the total solution space. Each chromosome chrom $_{i}$ is a two-dimensional matrix which consists of group number and tolerance width proportion. According to the ball-bearing assembly which is shown in Fig. 1, the proposed initial population structure is shown in Table IV.

Table IV: Structure of initial population in parts selective assembly scheme.

\begin{tabular}{|c|c|c|}
\hline Chromosome no. & Substring no. & Gene sequence \\
\hline \multirow[t]{3}{*}{1} & 1 & $4.1263-1.1818-5.0599-6.2087-2.2970-3.1264$ \\
\hline & 2 & $5.1050-3.1159-1.2577-2.3182-4.0222-6.1810$ \\
\hline & 3 & $6.1571-4.1222-2.2079-3.2181-5.0742-1.2204$ \\
\hline \multirow[t]{3}{*}{2} & 1 & $1.0820-3.0934-4.1638-5.3784-6.2237-2.0587$ \\
\hline & 2 & $4.1474-2.1345-3.2179-5.2137-6.1652-1.1213$ \\
\hline & 3 & $5.1152-6.3827-4.0039-3.2666-2.1308-1.1008$ \\
\hline \multirow[t]{3}{*}{3} & 1 & $3.0442-2.2695-6.3189-1.1012-4.1460-5.1202$ \\
\hline & 2 & $5.0357-2.0447-4.0231-6.4542-3.2326-1.2098$ \\
\hline & 3 & $3.2521-2.0858-6.0200-1.2622-4.0565-5.3235$ \\
\hline$\vdots$ & $\vdots$ & $\vdots$ \\
\hline \multirow[t]{3}{*}{10} & 1 & $2.2456-1.0782-5.1439-6.3692-3.1414-4.0217$ \\
\hline & 2 & $1.2385-6.0829-2.2593-3.0537-4.2601-5.1055$ \\
\hline & 3 & $1.3138-4.1265-2.2215-5.2329-6.0029-3.1025$ \\
\hline
\end{tabular}




\subsection{Evaluation module}

The objective criterion of the proposed model is to maximise the assembly acceptance rate, i.e., to improve the proportion of the quantity of assembled products that meet the assembly precision requirement. Considering the effects of two factors: (1) the difference on the number of parts in three matching groups, (2) the overlap between the assembly tolerance variation and the required assembly tolerance range, the calculation process for assembly acceptance rate of the $i^{\text {th }}$ combination in the $j^{\text {th }}$ chromosome includes the following two procedures:

(1) Calculate the maximum proportion of parts to be assembled.

(2) Calculate the proportion of qualified assembly tolerance variation to assembly tolerance variation.

The calculation for the maximum proportion of parts to be assembled is presented in Eq. (9):

$$
e_{i, x}=\min \left(e a_{j, i}, e b_{j, i}, e c_{j, i}\right)
$$

Where $e a, e b$ and $e c$ represent the proportion of part A, part $\mathrm{B}$ and part $\mathrm{C}$ in a volume, respectively.

The calculation for the assembly tolerance variation is given in Eqs. (10) to (12):

$$
\begin{gathered}
t_{\text {min }}=d_{1 \text { min }}+d_{2 \text { min }}+\cdots+d_{k-1 \text { min }}-\left(d_{k \text { max }}+d_{k+1 \text { max }}+\cdots+d_{\text {m max }}\right) \\
t_{\text {max }}=d_{1 \text { max }}+d_{2 \text { max }}+\cdots+d_{k-1 \text { max }}-\left(d_{k \text { min }}+d_{k+1 \text { min }}+\cdots+d_{\text {m min }}\right) \\
t_{\sigma}=\left[t_{\text {min }}, t_{\text {max }}\right]
\end{gathered}
$$

where $t_{\sigma}$ is the assembly tolerance variation, $1 \sim k-1$ represent increasing link, $k \sim m$ represent decreasing link, $d_{\min }$ represents the minimum dimension in a selected group, $d_{\max }$ represents the maximum dimension in a selected group.

The proportion function of qualified assembly tolerance variation is shown in Eq. (13):

$$
f_{j, i}=\left\{\begin{array}{l}
0, t_{\sigma} \nsubseteq T_{\sigma} \\
1, t_{\sigma} \subseteq T_{\sigma} \\
q, t_{\sigma} \nsubseteq T_{\sigma} \text { and } t_{\sigma} \cap T_{\sigma} \neq \varnothing
\end{array}\right.
$$

where $T_{\sigma}$ represents assembly precision requirement; $t_{\sigma} \nsubseteq T_{\sigma}$ represents that the clearance variation is beyond the required tolerance range, i.e., $t_{\max }<T_{\min }$ or $t_{\min }>T_{\max } ; t_{\sigma} \subseteq T_{\sigma}$ represents that the clearance variation is within the required tolerance range, i.e., $t_{\max } \leq$ $T_{\text {max }}$ and $t_{\text {min }} \geq T_{\text {min }} ; t_{\sigma} \nsubseteq T_{\sigma}$ and $t_{\sigma} \cap T_{\sigma} \neq \varnothing$ represent that the clearance variation is partly within the required tolerance range, i.e., $t_{\min }<T_{\min }$ and $t_{\max }>T_{\max }$, or $T_{\min } \leq$ $t_{\min } \leq T_{\max }$ and $t_{\max }>T_{\max }$, or $T_{\min } \leq t_{\max } \leq T_{\max }$ and $t_{\min }<T_{\min }$.

After grouping, the dimensions of parts in each group are approximate average distributions, thus $q$ can be obtained by calculations in Eq. (14):

where $0<q<1$.

$$
q=\frac{t_{\sigma} \cap T_{\sigma}}{t_{\sigma}}
$$

Consequently, the average acceptance rate of $k$ combinations in the $j^{\text {th }}$ chromosome represents the fitness value of the $j^{\text {th }}$ chromosome, and the corresponding fitness function is shown in Eq. (15):

$$
F_{i t}=\frac{\sum_{i=1}^{k} e_{j, i} f_{j, i}}{k}
$$

The fitness values of all chromosomes in the initial population are shown in Table V. 
Wang, Li, He, Tong: Modelling and Optimization for a Selective Assembly Process of Parts ...

Table V: The fitness values of all chromosomes in the initial population.

\begin{tabular}{|l|c|c|c|c|c|c|c|c|c|c|}
\hline Chromosome & 1 & 2 & 3 & 4 & 5 & 6 & 7 & 8 & 9 & 10 \\
\hline Fitness value & 0.235 & 0.198 & 0.225 & 0.205 & 0.229 & 0.335 & 0.189 & 0.171 & 0.253 & 0.265 \\
\hline
\end{tabular}

\subsection{New population generation module}

\section{Selection operator}

The roulette wheel mechanism is used in the selection operator. In the traditional selection operator, the probability of selecting each chromosome is concerned with the proportion of a fitness value in total fitness value, and the individual with the highest fitness value spreads rapidly and accounts for a large percent of population. Hence, the premature and local convergence will occur. In order to solve the above problems, a rank-based fitness assignment (RFA) method which has better robustness is applied. In RFA, the population is ranked based on the actual objective function value. The fitness depends solely on the rank position of the chromosome in the population. The selection process with RFA is outlined below:

(1) All the individuals are ranked in the ascending order according to the fitness value. The latter individual which has higher fitness value in the sequence is selected with a higher probability. The selection probability $P_{j}$ and the cumulative probability $F_{j}$ of the $j^{\text {th }}$ chromosome are given in Eq. (16) and Eq. (17), respectively. $N$ represents the population size, i.e. the number of chromosomes:

$$
\begin{gathered}
P_{j}=\frac{i}{\sum_{j=1}^{N} j}=\frac{2 j}{N(N+1)} \\
F_{j}=\sum_{x=1}^{j} P_{x}=\frac{j(j+1)}{N(N+1)}
\end{gathered}
$$

(2) A random number $r$ is generated from 0 to 1 , the $j^{\text {th }}$ individual will be selected as long as $F_{j-1}<r \leq F_{j}$.

(3) Repeat (2) for many times until $N$ individuals are selected to build the intermediate population which is the initial population of crossover operation in the next step. It is shown in Table VI.

Table VI: Construction of the intermediate population.

\begin{tabular}{|c|c|c|c|c|c|}
\hline $\begin{array}{c}\text { Rank } \\
\text { no. }\end{array}$ & $\begin{array}{c}\text { Chromosome } \\
\text { no. }\end{array}$ & $\begin{array}{c}\text { Selection } \\
\text { probability }\end{array}$ & $\begin{array}{c}\text { Cumulative } \\
\text { probability }\end{array}$ & $\begin{array}{c}\text { Random } \\
\text { number }\end{array}$ & $\begin{array}{c}\text { Individual } \\
\text { selected }\end{array}$ \\
\hline 1 & 6 & 0.0182 & 0.0182 & 0.1403 & 10 \\
\hline 2 & 9 & 0.0364 & 0.0545 & 0.2601 & 7 \\
\hline 3 & 3 & 0.0545 & 0.1091 & 0.0868 & 3 \\
\hline 4 & 10 & 0.0727 & 0.1818 & 0.4294 & 5 \\
\hline 5 & 7 & 0.0909 & 0.2727 & 0.2573 & 7 \\
\hline 6 & 8 & 0.1091 & 0.3818 & 0.2976 & 8 \\
\hline 7 & 5 & 0.1273 & 0.5091 & 0.4249 & 5 \\
\hline 8 & 4 & 0.1455 & 0.6545 & 0.1192 & 10 \\
\hline 9 & 1 & 0.1636 & 0.8182 & 0.9394 & 2 \\
\hline 10 & 2 & 0.1818 & 1 & 0.7064 & 1 \\
\hline
\end{tabular}

\section{Crossover operator}

The crossover operator exchanges part genes of two parent chromosomes obtained by the selection operator to form two new chromosomes. Without consideration of the individual fitness in this stage, it is necessary to meet constrains defined in Eq. (7) to ensure that the generated solution is feasible. Hence, adjustments have to be made on the chromosomes generated by the crossover operator. The crossover procedure consists of the following steps. 
(1) In the intermediate population constructed by the selection operator, a pair of chromosomes is selected as the parents for crossover.

(2) The crossover probability $P_{c}$ is set to 0.9 , then a random number $r$ is generated from 0 to 1 , then $r$ is compared with $P_{c}$. If $r<P_{c}$, then go to (3) to carry out crossover operation, otherwise back to (1) to select another pair of chromosomes.

(3) Using a single point crossover method between the substrings of the selected pair of chromosomes, one out of $m$ substrings is selected randomly for crossover operation. The position of crossover point is identified through generating a random integer between 1 and $k$ (the number of groups).

(4) Duplicate the genes before the position of crossover point in the crossover substring Parent 1 (in the parent chromosome Parent I) and the crossover substring Parent 2 (in the parent chromosome Parent II) into the intermediate substring Copy 1 and Copy 2, respectively.

(5) Compare the group number genes $\left(b_{i, k}\right)$ of Parent 2 with Copy 1, and remove the same genes from Parent 2, i.e., all the group number genes of Copy 1, are removed from Parent 2. Afterwards, the grouping tolerance relative width genes $\left(a_{i, k}\right)$ in the left gene section of Parent 2 need a reallocation. Finally, combine the left gene section of Parent 2 with the intermediate substring Copy 1, to generate a new substring Offspring 1 of the offspring chromosome Offspring I. The same operations can also be implemented to generate a new substring Offspring 2 of the offspring chromosome Offspring II.

An example of the crossover operator between two parent chromosomes is tabulated in Table VII. In this illustrated example, the fourth gene of the second substring is randomly chosen to be the crossover point.

Table VII: Example of the crossover operator.

\begin{tabular}{|c|c|c|c|}
\hline Operation & & Substring & Gene sequence \\
\hline Before crossover & \multirow{3}{*}{ Parent I } & 1 & $2.2456-1.0782-5.1439-6.3692-3.1414-4.0217$ \\
\cline { 3 - 4 } & & $\mathbf{2}$ & $1.2385-6.0829-2.2593-\mathbf{3 . 0 5 3 7 - 4 . 2 6 0 1 - 5 . 1 0 5 5}$ \\
\cline { 3 - 4 } & & 3 & $1.3138-4.1265-2.2215-5.2329-6.0029-3.1025$ \\
\cline { 3 - 4 } & \multirow{3}{*}{ Parent II } & 1 & $1.0820-3.0934-4.1638-5.3784-6.2237-2.0587$ \\
\cline { 3 - 4 } & & $\mathbf{2}$ & $4.1474-2.1345-3.2179-\mathbf{5 . 2 1 3 7 - 6 . 1 6 5 2 - 1 . 1 2 1 3}$ \\
\cline { 3 - 4 } & & 3 & $5.1152-6.3827-4.0039-3.2666-2.1308-1.1008$ \\
\hline \multirow{3}{*}{ After crossover } & \multirow{3}{*}{ Offspring I } & 1 & $2.2456-1.0782-5.1439-6.3692-3.1414-4.0217$ \\
\cline { 3 - 4 } & & $\mathbf{2}$ & $1.2385-6.0829-2.2593-\mathbf{4 . 1 8 4 9 - 3 . 2 0 5 6 - 5 . 0 2 8 8}$ \\
\cline { 3 - 4 } & \multirow{3}{*}{ Offspring II } & 3 & $1.3138-4.1265-2.2215-5.2329-6.0029-3.1025$ \\
\cline { 3 - 4 } & & $\mathbf{2}$ & $1.0820-3.0934-4.1638-5.3784-6.2237-2.0587$ \\
\cline { 3 - 4 } & & 3 & $5.1474-2.1345-3.2179-\mathbf{1 . 2 7 8 0 - 6 . 1 9 2 5 - 5 . 0 2 9 7}$ \\
\cline { 3 - 4 } & & &
\end{tabular}

\section{Mutation operator}

The mutation operator conducts random transforms on the new offspring individuals to keep population's diversity. It enlarges the search space of GA to avoid achieving a premature convergence of local optimal solution. In mutation, the mutation operation is performed for all the genes of each new offspring individual with a small probability $P_{m}$, as described in the following procedures.

(1) The mutation probability $P_{m}$ is se to 0.05 , then a random number $r$ is generated from 0 to 1 , then $r$ is compared with $P_{c}$ If $r<P_{m}$, then go to (2) to perform mutation operation, otherwise back to (1) to judge the next gene.

(2) In the mutation operation, it is necessary to meet constrains defined in Eq. (7) to ensure that the generated solution is feasible. The group number gene $\left(b_{i, k}\right)$ in the selected gene is exchanged with the nearest and different gene, and the grouping tolerance relative width genes $\left(a_{i, k}\right)$ of these two genes are reallocated. 
An example of the mutation operator is tabulated in Table VIII, in which the genes in a big bold font are muted.

Table VIII: Example of the mutation operator.

\begin{tabular}{|c|c|c|c|}
\hline Operation & & Substring & Gene sequence \\
\hline Before mutation & Parent & 1 & $2.2456-1.0782-5.1439-6.3692-3.1414-4.0217$ \\
\cline { 3 - 4 } & & 2 & $1.2385-6.0829-2.2593-4.1849-3.2056-5.0288$ \\
\cline { 3 - 4 } & & 3 & $1.3138-4.1265-2.2215-5.2329-6.0029-3.1025$ \\
\hline \multirow{2}{*}{ After mutation } & \multirow{2}{*}{ Offspring } & 1 & $1.2456-2.1072-5.1149-6.3692-3.1414-4.0217$ \\
\cline { 3 - 4 } & & 2 & $1.2385-6.0829-2.2593-4.1849-3.2056-5.0288$ \\
\cline { 3 - 4 } & & 3 & $1.3138-2.1664-4.1816-5.2329-6.0029-3.1025$ \\
\hline
\end{tabular}

\section{Elitist operator}

In the evolutionary process of GA, the phenomenon, that the optimal individual structure is possible to be destroyed and lost without the protection on the current optimal individual, will occur repeatedly. To solve the above problem, we adopt the elitist strategy. As a function feature of GA, the elitist strategy provides a means to reduce genetic drift. This strategy permits the best individual to pass/copy their attributes into the next generation. The use of elitist strategy with GA is explained in detail by Ahn and Ramakrishna [24].

In this work, the specific operation of elitist strategy is that, take out the best individual in the population, then copy the best individual to replace the individual with the least fitness value in the next generation. By the implementation of elitist strategy, the optimal individual is preserved to improve the evolutionary efficiency and the global convergence ability.

\subsection{Termination}

The GA will be terminated when a pre-set number of iterations is reached. After many experiments, the iteration number is set as 10000 .

\subsection{Output module}

As the output, the optimal solution of assembly scheme is given in each iteration. The maximum assembly acceptance rate and the best assembly scheme are shown in Table IX.

Table IX: Best assembly scheme and its assembly acceptance rate.

\begin{tabular}{|c|c|c|}
\hline Part & Assembly scheme & Assembly acceptance rate (\%) \\
\hline A & $4.1677-5.1387-6.1806-3.0841-2.1938-1.2352$ & \\
\hline B & $3.2654-4.2136-6.1605-5.2702-2.0136-1.0767$ & \multirow{2}{*}{73.84} \\
\hline C & $4.1716-3.1382-5.1436-2.0908-6.4509-1.0048$ & \\
\hline
\end{tabular}

\section{NUMERICAL EXPERIMENTS}

To search the optimal assembly scheme in the ball-bearing assembly process, an improved GA which is based on the developed model is encoded in MATLAB R2014a and the computer simulation experiments are performed on a laptop with $2.2 \mathrm{GHz} \mathrm{CPU}$ and $8 \mathrm{~GB}$ RAM. To prove the advantage of the proposed method, some numerical experiments are designed and conducted in three schemes, which are shown as follows:

(1) Traditional grouping selective assembly scheme,

(2) Sequential assembly scheme,

(3) Proposed grouping selective assembly scheme with improved GA.

As described in the previous sections, the experimental parameters for three schemes are identical. For comparison, the obtained maximum assembly acceptance rates for three cases are tabulated in Table X. The evolution performance for the improved GA is shown in Fig. 3. 
Considering that the grouping tolerance relative width which is generated in each iteration is random and variable, the obtained optimal assembly scheme and its corresponding acceptance rate are not sole. Nevertheless, with regard to assembly acceptance rate under any parameters, it is found that the proposed grouping selective assembly scheme with improved GA is better than the traditional grouping selective assembly scheme and the sequential assembly scheme with more than $50 \%$ higher, respectively. In addition, the proposed algorithm is found to be cost-effective for different volume sizes.

Table X: The assembly acceptance rates obtained for three cases.

\begin{tabular}{|l|c|}
\hline \multicolumn{1}{|c|}{ Scheme name } & Assembly acceptance rate (\%) \\
\hline Traditional grouping selective assembly scheme & 35.33 \\
\hline Sequential assembly scheme & 49 \\
\hline Proposed grouping selective assembly scheme & 73.84 \\
\hline
\end{tabular}

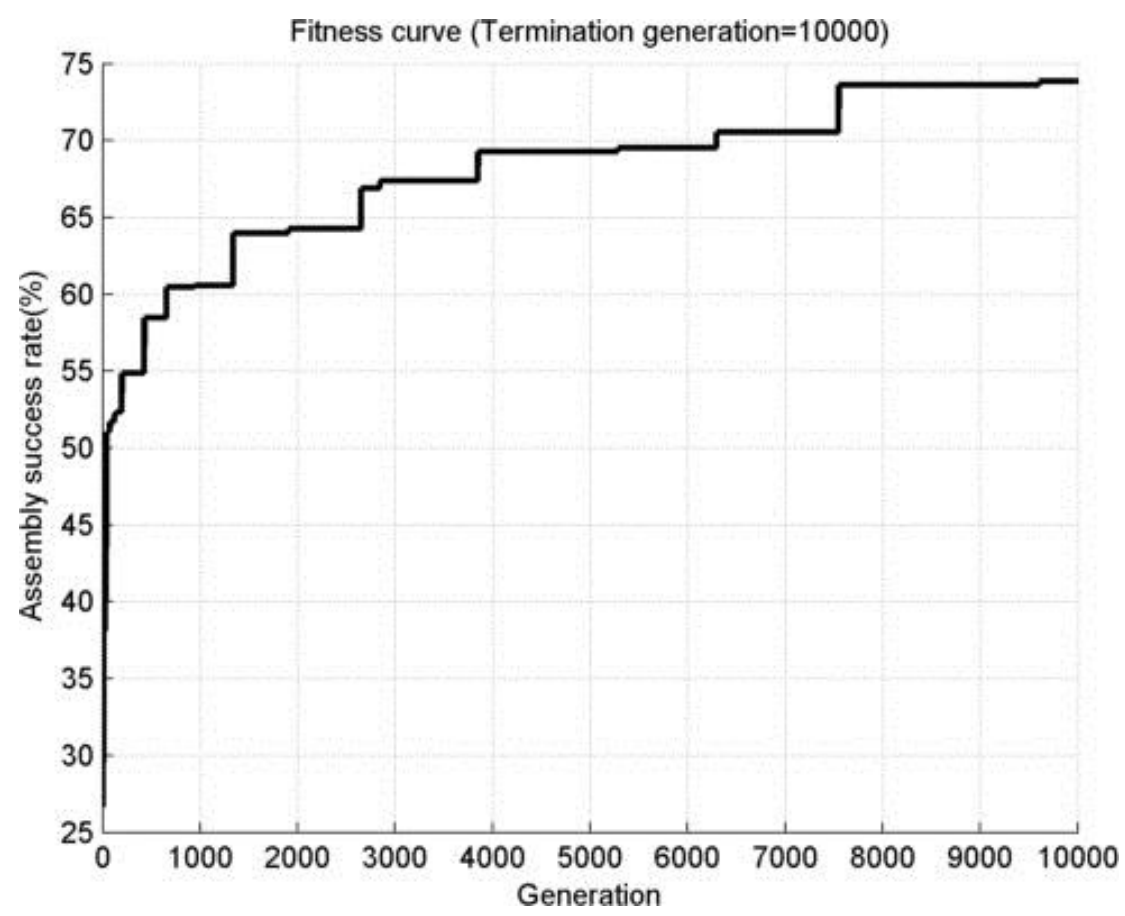

Figure 3: Evolution performance for the improved GA.

\section{CONCLUSION}

This research work makes an attempt to study the optimization problem of grouping selective assembly scheme in a ball-bearing assembly process of parts with non-normal distribution. With the current capabilities of advanced dimension measurement apparatus and highperformance computers, a new grouping selective assembly scheme is proposed instead of traditional grouping selective assembly scheme. A modified GA has been developed to enhance the assembly acceptance rate by reducing the surplus parts.

In comparison to the traditional grouping selective assembly scheme, the numerical experiments results illustrate that that the proposed grouping selective assembly scheme with improved GA is able to raise the percentage of acceptable assembled products from $35.33 \%$ to $73.84 \%$. By applying the optimization and simulation model, a ball-bearing assembly as illustrated in section 3.1 can be produced in a more efficient manner than it does commonly in traditional methods. It is also found that the proposed method is effective and feasible irrespective of the volume size of assembled products produced. 


\section{ACKNOWLEDGEMENT}

This work was financially supported by the National Defense Science and Technology Project Foundation (No. 0106142), and MOE (Ministry of Education in China) Project of Humanities and Social Sciences (No. 17YJC630139). The supports are gratefully acknowledged.

\section{REFERENCES}

[1] Lanza, G.; Haefner, B.; Kraemer, A. (2015). Optimization of selective assembly and adaptive manufacturing by means of cyber-physical system based matching, CIRP Annals Manufacturing Technology, Vol. 64, No. 1, 399-402, doi:10.1016/j.cirp.2015.04.123

[2] Lochte, C.; Kayasa, J.; Herrmann, C.; Raatz, A. (2012). Methods for implementing compensation strategies in micro production systems supported by a simulation approach, $6^{\text {th }}$ International Precision Assembly Technologies ans Systems Seminar, 118-125, doi:10.1007/978-3-642-28163$\underline{115}$

[3] Xu, H.-Y.; Kuo, S.-H.; Tsai, J. W. H.; Ying, J. F.; Lee, G. K. K. (2014). A selective assembly strategy to improve the components' utilization rate with an application to hard disk drives, International Journal of Advanced Manufacturing Technology, Vol. 75, No. 1-4, 247-255, doi:10.1007/s00170-014-6106-y

[4] Yang, S.; Wang, H.; Hu, S. J.; Lin, Y.-t. (2013). Modeling assembly systems with repetitive operations, CIRP Annals - Manufacturing Technology, Vol. 62, No. 1, 5-8, doi:10.1016/j.cirp. 2013.03.006

[5] Schmitt, J.; Raatz, A.; Dietrich, F.; Dröder, K.; Hesselbach, J. (2014). Process and performance optimization by selective assembly of battery electrodes, CIRP Annals - Manufacturing Technology, Vol. 63, No. 1, 9-12, doi:10.1016/j.cirp.2014.03.018

[6] Huang, W.; Ceglarek, D. (2002). Mode-based decomposition of part form error by discretecosine-transform with implementation to assembly and stamping system with compliant parts, CIRP Annals - Manufacturing Technology, Vol. 51, No. 1, 21-26, doi:10.1016/s00078506(07)61457-7

[7] Fang, X. D.; Zhang, Y. (1995). A new algorithm for minimising the surplus parts in selective assembly, Computers \& Industrial Engineering, Vol. 28, No. 2, 341-350, doi:10.1016/03608352(94)00183-N

[8] Kumar, M. S.; Kannan, S. M.; Jayabalan, V. (2007). A new algorithm for minimizing surplus parts in selective assembly by using genetic algorithm, International Journal of Production Research, Vol. 45, No. 20, 4793-4822, doi:10.1080/00207540600810085

[9] Matsuura, S.; Shinozaki, N. (2011). Optimal process design in selective assembly when components with smaller variance are manufactured at three shifted means, International Journal of Production Research, Vol. 49, No. 3, 869-882, doi:10.1080/00207541003604851

[10] Raj, M. V.; Sankar, S. S.; Ponnambalam, S. G. (2011). Genetic algorithm to optimize manufacturing system efficiency in batch selective assembly, International Journal of Advanced Manufacturing Technology, Vol. 57, No. 5-8, 795-810, doi:10.1007/s00170-011-3326-2

[11] Lu, C.; Fei, J.-F. (2015). An approach to minimizing surplus parts in selective assembly with genetic algorithm, Proceedings of the Institution of Mechanical Engineers, Part B: Journal of Engineering Manufacture, Vol. 229, No. 3, 508-520, doi:10.1177/0954405414530896

[12] Mease, D.; Nair, V. N.; Sudjianto, A. (2004). Selective assembly in manufacturing: Statistical issues and optimal binning strategies, Technometrics, Vol. 46, No. 2, 165-175, doi: $\underline{10.1198 / 004017004000000185}$

[13] Kannan, S. M.; Sivasubramanian, R.; Jayabalan, V. (2009). Particle swarm optimization for minimizing assembly variation in selective assembly, International Journal of Advanced Manufacturing Technology, Vol. 42, No. 7-8, 793-803, doi:10.1007/s00170-008-1638-7

[14] Wang, W.; Li, D.; Chen, J. (2011). Minimizing assembly variation in selective assembly for complex assemblies using genetic algorithm, $2^{\text {nd }}$ International Conference on Mechanic Automation and Control Engineering, 1401-1406, doi:10.1109/MACE.2011.5987207

[15] Raj, M. V.; Sankar, S. S.; Ponnambalam, S. G. (2011). Optimization of assembly tolerance variation and manufacturing system efficiency by using genetic algorithm in batch selective 
assembly, International Journal of Advanced Manufacturing Technology, Vol. 55, No. 9-12, 1193-1208, doi:10.1007/s00170-010-3124-2

[16] Osma, A. (2009). A statistical approach to comparing wheel hub forging processes, Proceedings of the Institution of Mechanical Engineers, Part D: Journal of Automobile Engineering, Vol. 223, No. 12, 1559-1576, doi:10.1243/09544070JAUTO1207

[17] Raj, M. V.; Sankar, S. S.; Ponnambalam, S. G. (2012). Particle swarm optimization algorithm to maximize assembly efficiency, International Journal of Advanced Manufacturing Technology, Vol. 59, No. 5-8, 719-736, doi:10.1007/s00170-011-3512-2

[18] Tang, M.; Gong, D.; Liu, S.; Zhang, H. (2016). Applying multi-phase particle swarm optimization to solve bulk cargo port scheduling problem, Advances in Production Engineering \& Management, Vol. 11, No. 4, 299-310, doi:10.14743/apem2016.4.228

[19] Gong, D.; Tang, M.; Liu, S.; Li, Q. (2017). Reconsidering production coordination: A principalagent theory-based analysis, Advances in Production Engineering \& Management, Vol. 12, No. 1, 51-61, doi:10.14743/apem2017.1.239

[20] Tang, M.; Gong, D.; Liu, S.; Lu, X. (2017). Finding key factors affecting the locations of electric vehicle charging stations: A simulation and ANOVA approach, International Journal of Simulation Modelling, Vol. 16, No. 3, 541-554, doi:10.2507/IJSIMM16(3)CO15

[21] Wen, F.; He, Z.; Dai, Z.; Yang, X. (2014). Characteristics of investors' risk preference for stock markets, Economic Computation and Economic Cybernetics Studies and Research, Vol. 48, No. $3,235-254$

[22] Wen, F.; Gong, X.; Cai, S. (2016). Forecasting the volatility of crude oil futures using HAR-type models with structural breaks, Energy Economics, Vol. 59, 400-413, doi:10.1016/j.eneco. 2016.07.014

[23] Wen, F.; Xiao, J.; Huang, C.; Xia, X. (2018). Interaction between oil and US dollar exchange rate: nonlinear causality, time-varying influence and structural breaks in volatility, Applied Economics, Vol. 50, No. 3, 319-334, doi:10.1080/00036846.2017.1321838

[24] Ahn, C. W.; Ramakrishna, R. S. (2003). Elitism-based compact genetic algorithms, IEEE Transactions on Evolutionary Computation, Vol. 7, No. 4, 367-385, doi:10.1109/TEVC. $\underline{2003.814633}$ 